\title{
MODELLING OF MICROHARDNESS VALUES BY MEANS OF ARTIFICIAL NEURAL NETWORKS OF AL/SiC $P$ METAL MATRIX COMPOSITE MATERIAL COUPLES PROCESSED WITH DIFFUSION METHOD
}

\author{
Mustafa Taşkın and Uğur Çalıgülü \\ University of Firat, Faculty of Technical Education, Department of Metal, 23119 \\ Elazığg, Turkey. mtaskin@,firat.edu.tr
}

\begin{abstract}
In this study, modelling of microhardness values by means of artificial neural networks of $\mathrm{Al} / \mathrm{SiC}_{\mathrm{p}}$ metal matrix composite material couples with diffusion method and manufactured by powder metallurgy process, were obtained using a backpropagation neural network that uses gradient descent learning algorithm.

After diffusion bonding and relevant test, to prepare the training and test (checking) set of the network, results were recorded in a file on a computer. Then, the neural network was trained using the prepared training set. At the end of the training process, the test data were used to check the system accuracy. As a result the neural network was found successful in the prediction of modelling of microhardness values of $\mathrm{Al} / \mathrm{SiC}_{\mathrm{p}}$ metal matrix composite material couples processed with diffusion method and behavior.
\end{abstract}

Keywords-Artificial Neural Network, MMCs, Diffusion Bonding, Microhardness

\section{INTRODUCTION}

Aluminum-based, particulate-reinforced metal matrix composites (MMCs) are of concerns for structural carrying outs where weight saving is of primary concern. There are several production techniques to getting in manufacturing the MMC materials. An artificial neural network is a parallel-dispensed information proceeding system. It stores the specimens with dispensed coding, thus forming a trainable nonlinear system. The main idea of neural network draw near to resembles the human brain functioning. Given the inputs and longing outputs, it is also self-adaptive to the habitat so as to respond different inputs rationally. The neural network theory deals with learning from the preceding obtained data, which is named as training or learning set, and then to check the system accomplishment using test data [1-2]. Artificial Neural networks (ANNs) have been used to model the human vision system. They are biologically inspired and contain a large number of simple processing elements that perform in a manner analogous to the most elementary functions of neurons. Artificial neural networks learn by experience, generalize from previous experiences to new ones, and can make decisions. Neural elements of a human brain have a computing speed of a few milliseconds, whereas the computing speed of electronic circuits is on the order of microseconds. The ANNs are parallel process elements which has characteristic in below.

-ANN is a mathematical model of a biological neuron.

-ANN has very process elements which are related another.

-ANN keeps knowledge with connection weights. 
Neural network models provide an alternative approach to implementing enhancement techniques. A simple process element of the NNs is given in Fig.1. Output of $i_{\text {th }}$ process element at this simple model is given at Equation 1.

$y(t+1)=a\left(\sum_{j=1}^{m} w_{i j} x_{j}(t)-\theta_{i}\right)$

In there, $a$ is activation function, $\theta_{i}$ is threshold value of $i_{\text {th }}$ process element. Knowledge processes of process element compose from two parts: input and output. Output of $i_{\text {th }}$ process element is calculated with Equation 2 [3-4].

$$
\mathrm{f}_{\mathrm{i}} \triangleq \text { net }_{\mathrm{i}}=\sum_{\mathrm{j}=1}^{\mathrm{m}} \mathrm{w}_{\mathrm{ij}} \mathrm{x}_{\mathrm{j}}-\theta_{\mathrm{i}}
$$

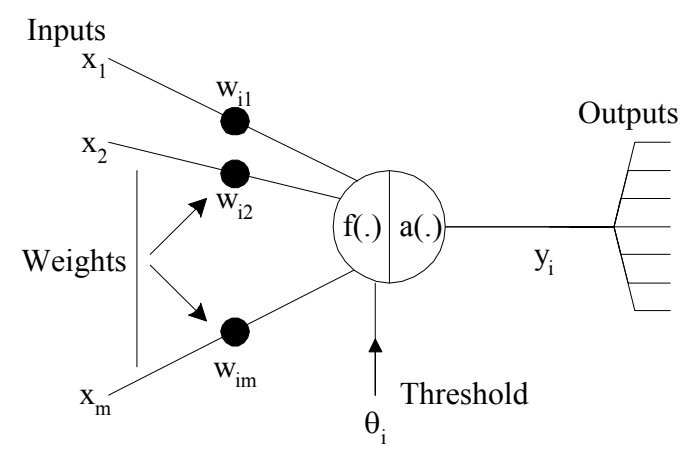

Figure 1. The Mathematical Model of Neuron

Neural networks were procured a basically different draw near to material modeling and material processing control techniques than statistical or numerical procedures. This method is feasible in many areas of engineering and has produced promising to prepare results in the areas of material modeling and proceduring. One of the main advantages of this approach is that there is no need to make a priori assumptions about material behavior although in more were sophisticated neural network modeling projects one may take advantage of the information of the procedure in network design. Even though multi-layered neural network models cannot make sure a global minimum solution for any given problem, it is a sensible assumpttions that if the network is trained on a extensive database with a suitable representation project, the resulting model will approximate all of the laws of mechanics that the actual material or process obeys [5-6].

Neural networks are essentially connectionist system, in which different nodes called neurons are interconnected. A typical neuron accepts one or more input signals and procures an output signal trusting in the proceduring function of the neuron. This output is conveyed to connected neurons in varying intensities, the signal intensity being decided by the weights. Feed forward networks are jointly used. A feed forward network has a consecutive of layers consisting of a number of neurons in each layer. The output of neurons of one layer come to exists input to neurons of the achieving 
layer. The first layer, called an input layer, accepts data from the outside world. The last layer is the output layer, which sends knowledge out to users. Layers that lie between the input and output layers are called hidden layers and have no direct touch with the environment. Their presence is needed in order to procure complexity to network architecture for modeling non-linear functional kinship. After choosing the network architecture, the network is tested by using back propagation algorithm, where back propagation algorithm is the productive optimization method used for underrating the error through weight arrangement The trained neural network has to be experimented by supplying testing data [7].

The basic fundamentals to build the system model on the basis of NN consist of:

(a) connecting the artificial neurons into a network with respect to certain rules and a topology;

(b) regulating the weights between neurons in term of an proofreading criterion;

(c) establishing the topology and free parameters of the NN by learning specimen data (input patterns) repeatedly;

(d) determining the system model by taking advantage of the strong learning ability of ANN (Fig. 2).

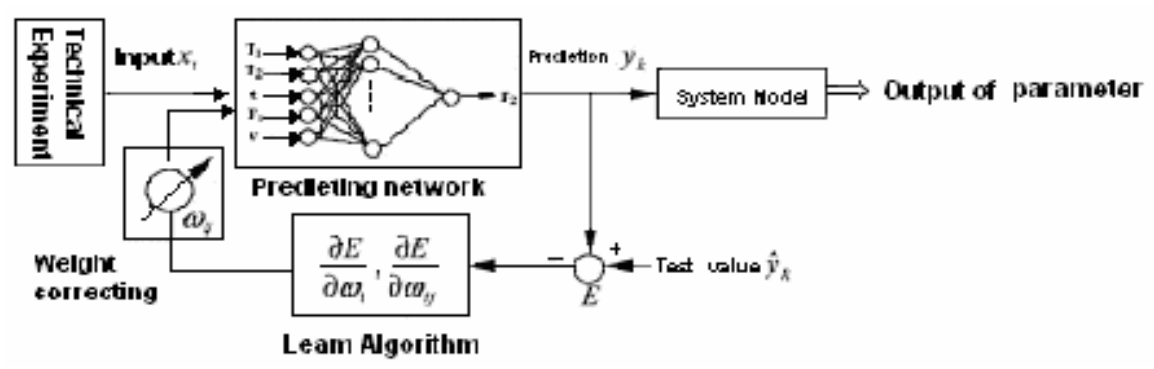

Figure 2. Scheme of Modelling The System by ANN [8].

The information included in the illustration data was acquired via the improved back propagation (BP) learning algorithm. The parameters of the BP network were defined as follows:

The input vectors $[\mathrm{X}=\mathrm{x} 0, \mathrm{x} 1, \ldots, \mathrm{xn} 1]^{\mathrm{T}}$

The output vectors $[\mathrm{Y}=\mathrm{y} 0, \mathrm{y} 1, \ldots, \mathrm{ym} 1]^{\mathrm{T}}$

where the symbols $\mathrm{n}, \mathrm{h}$ and $\mathrm{m}$ represented the number of neurons in the input layer, the hidden layer and the output layer, sequentially.

Joining of the powder metallurgy products $(\mathrm{P} / \mathrm{M})$ by diffusion bonding process is important both to protect the microstructural properties of parent materials and bonding behavior of joining materials [9]. Diffusion bonding is a solid state coalescence of contacting surfaces occur at a temperature below the melting point $\left(\mathrm{T}_{\mathrm{m}}\right)$ of the materials to be joined with the loads and the period, below those that would cause macro deformation and a significant properties change at the parent materials [10]. The process is depended on a number of parameters in particular, bonding temperature, atmosphere, time, pressure and surface roughness. Process pressure is selected high enough to dislocate the surface oxides. Bonding period should be selected long enough for the completion of the diffusion mechanism at the interface. Diffusion bonding is an 
advanced bonding process in which two materials, similar or dissimilar, can be bonded in solid state. Process temperature is selected as $0.5-0.7\left(\mathrm{~T}_{\mathrm{m}}\right)$. Super plasticity may occur at low temperatures [11-12].

\section{EXPERIMENTAL STUDIES}

SiC particulate Al alloy MMCs specimens to be produced by hot pressing method were fabricated by powder metallurgy process. $1 \% \mathrm{Mg}, 3 \% \mathrm{Si}$ powders were mixed with $99 \%$ Al. SiC particles with a $42 \mu \mathrm{m}$ mean diameter were added to the matrix at 5, 10, 20 (wt) \% fractions. Powders were properly mixed with mechanic mixers for homogeneity of the formation. The mixture was cold compacted at $400 \mathrm{MPa}$ in the $\phi 12 \times 60 \mathrm{~mm}$ steel dies. This is followed by sintering at $600{ }^{\circ} \mathrm{C}$ in argon atmosphere for 30 minutes. Finally the specimens were hot compacted and extruded at $200 \mathrm{MPa}$ pressure. Mixture rations and density of specimens were presented in Table 1.

Table 1. Specimens of Density and Mixture Rations Values

$\begin{array}{cccccc}\text { Sample } & \% \text { Al } & \text { \%Mg } & \text { \%Si } & \text { \%SiC } & \text { Density }\left(\mathbf{g r} / \mathbf{c m}^{3}\right) \\ \text { N 1 } & 91 & 1 & 3 & 5 & 2.69 \\ \text { N 2 } & 86 & 1 & 3 & 10 & 2.72 \\ \text { N 3 } & 81 & 1 & 3 & 20 & 2.76\end{array}$

Work pieces were prepared for diffusion bonding and surfaces to be joined were protected against corrosion and oxidation. Al alloy MMC specimens with 5-5, 5-10, $5-20,10-10,10-20$ ve $20-20 \% \mathrm{SiC}$ (wt) fractions were coupled and bonded at diffusion bonding apparatus. Schematic illustration of diffusion bonding apparatus is given in Fig. 3. Diffusion bondings were performed at 550, 575, 600 and $625^{\circ} \mathrm{C}$ process temperatures and for 20, 40 and 60 min periods under $0.25 \mathrm{MPa}$ constant pressure.

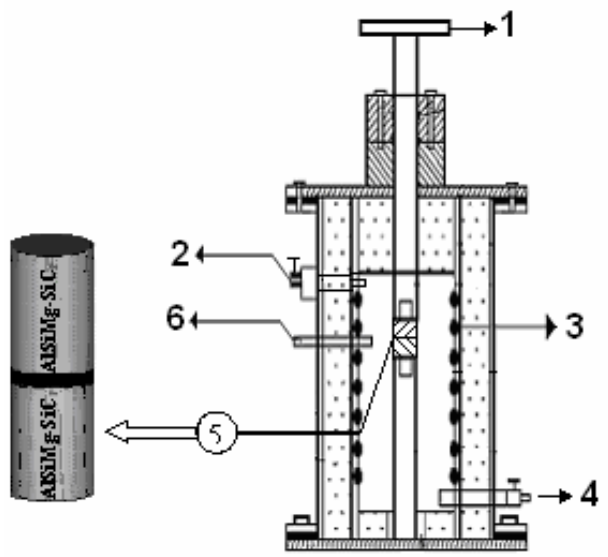

1-Load 2-Argon Outlet 3-Heat Coil 4-Argon Inlet 5-Specimens 6-Thermocouple

Figure 3. Schematic İllustration of Diffusion Bonding Apparatus

After the bonding process, specimens were tested for microhardness. Specimens were cut perpendicular to the bonding interface to facilitate longitudinal microstructure 
cross section examinations. Micro hardness values were measured at interface and it's both side by Hv scale under a load of 100 gr. (Fig. 4). Grinding of the surface were followed by etching with Keller etchant. Metallographic evaluations and investigations were made by the aid of optical microscopy and SEM.

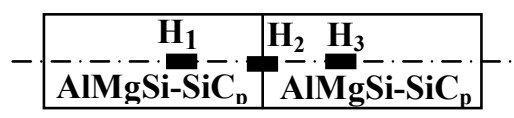

Figure 4. Schematic İllustraion of Microhardness Measuremend of Specimens

Modeling of microhardness values of diffusion bonding behavior at MATLAB program diffusion bonding period, process temperature and $\mathrm{SiC}_{\mathrm{p}}$ reinforcement (weight) fractions were employed as input and microhardness of the bonded interfaces were recorded as output parameters. Back propagation Multilayer Perceptron (MLP) ANN were used for training of experimental results. ANN modeling the microhardness of the interface of diffusion bonded composites were carried out with the aid of ANN block diagram given at Fig. 5. MLP architecture and training parameters were presented in Table 2.

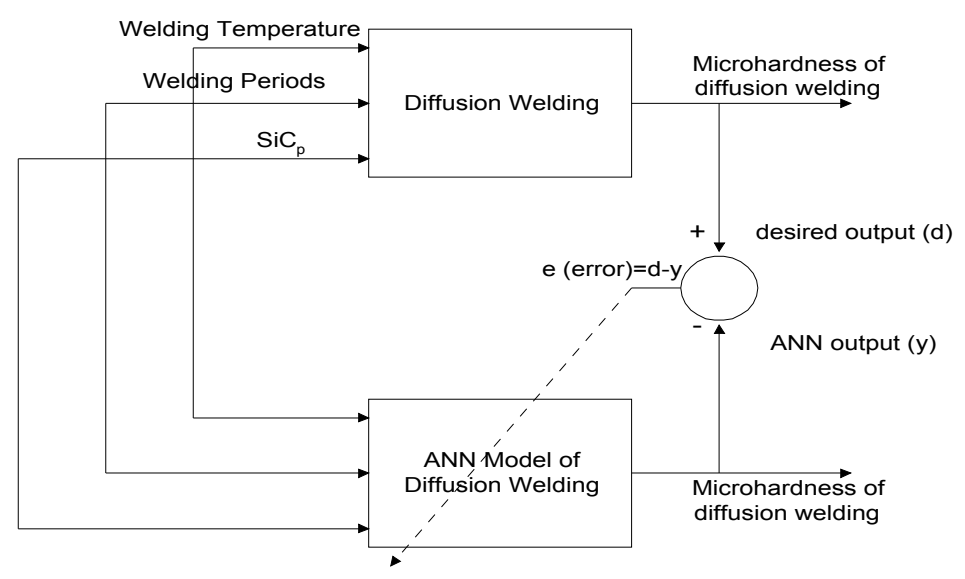

Figure 5. Block diagram of the ANN

Table 2. MLP Architecture and Training Parameters

The number of layers

The number of neuron on the layers

The initial weights and biases

Activation functions

Training parameters Learning rule

Adaptive learning rate

Momentum constant

Sum-squared error
3

Input: 3, Hidden: 10, Output: 1

The Nguyen-Widrow method

Log-sigmoid

Back-propagation

Initial: 0.0001 Increase: 1.05 Decrease: 0.7

0.98

0.0000001 


\section{RESULTS AND DISCUSSION}

Deformation of surface asperities by plastic flow and creep, grain boundary diffusion of atoms to the voids and grain boundary migration, volume diffusion of atoms to voids can be listed as a sequence of metallurgical stages of the diffusion bonding. Especially with aluminum alloys diffusion bonding can be achieved with adherent surface oxides. In general, the oxide is not removed, but is simply dispersed over a greater surface area in an enclosed environment, in which oxidation cannot recur.

At elevated temperatures diffusion mechanism were accelerated and diffusion period were decreased to achieve the same coalescence. Relatively poor coalescence were achieved at $550-575{ }^{\circ} \mathrm{C}$ process temperatures. Voids were traced at the bond interface. SEM micrographs of weld specimens bonded at $550-575{ }^{\circ} \mathrm{C}$ process temperatures presented at Fig. 6 and 7.
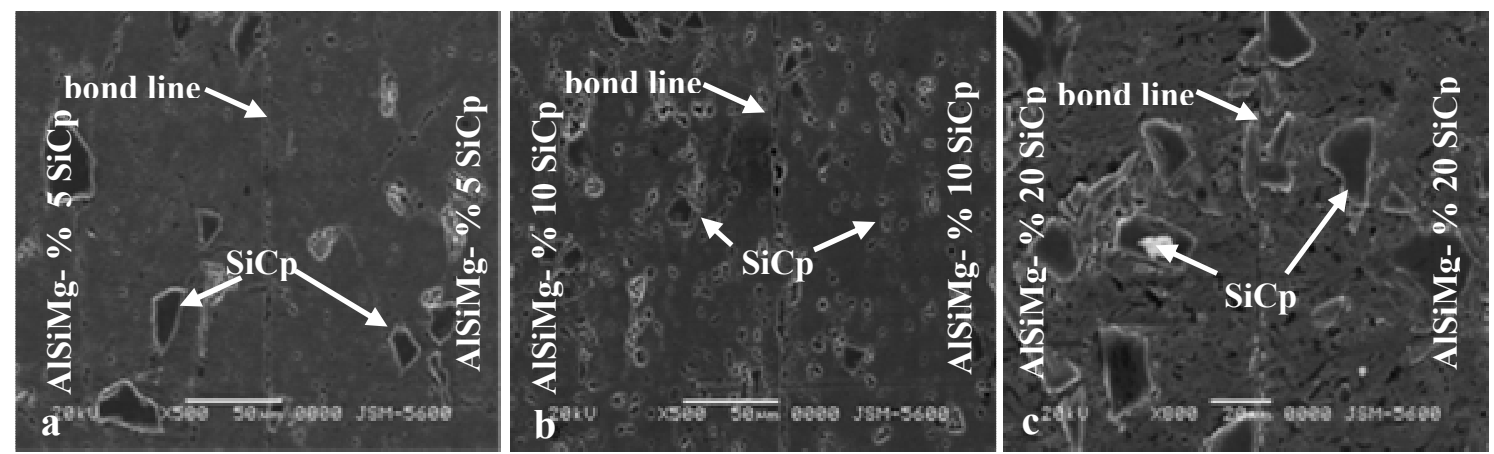

Figure 6. SEM Micrograph of Specimens Bonded at $550^{\circ} \mathrm{C}$ Process Temp. for a-20, b-40, c-60 min.
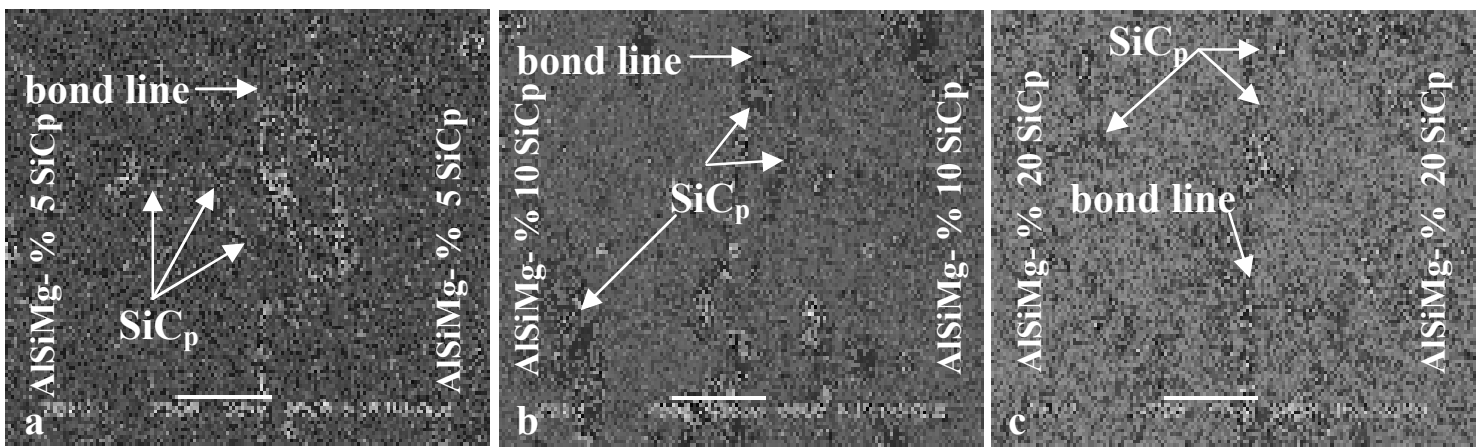

Figure 7. SEM Micrograph of Specimens Bonded at $575^{\circ} \mathrm{C}$ Process Temp. for a-20, b-40, c-60 min

Reliable coalescence were achieved at weld specimens bonded at $600-625{ }^{\circ} \mathrm{C}$. The interface were free of voids and were nearly indistinguishable from parent materials. The mean microhardness results were relatively higher than the other process 
temperatures. SEM micrographs of weld specimens bonded at $600-625{ }^{\circ} \mathrm{C}$ process temperatures were presented at Fig.8 and 9.
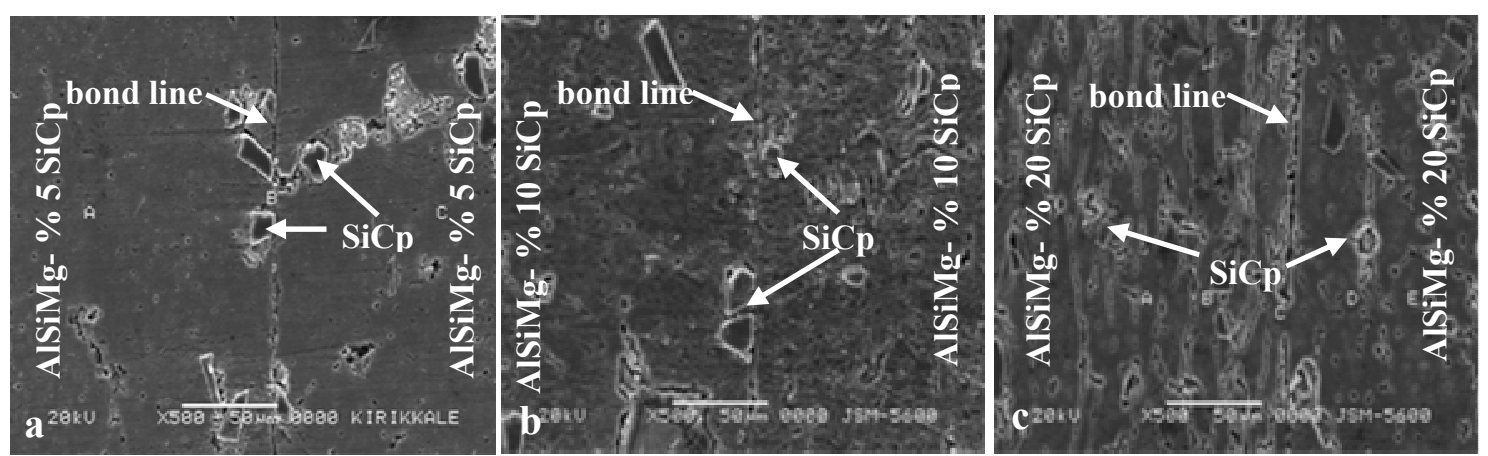

Figure 8. SEM Micrograph of Specimens Bonded at $600{ }^{\circ} \mathrm{C}$ Process Temp. for a-20, b-40, c-60 min
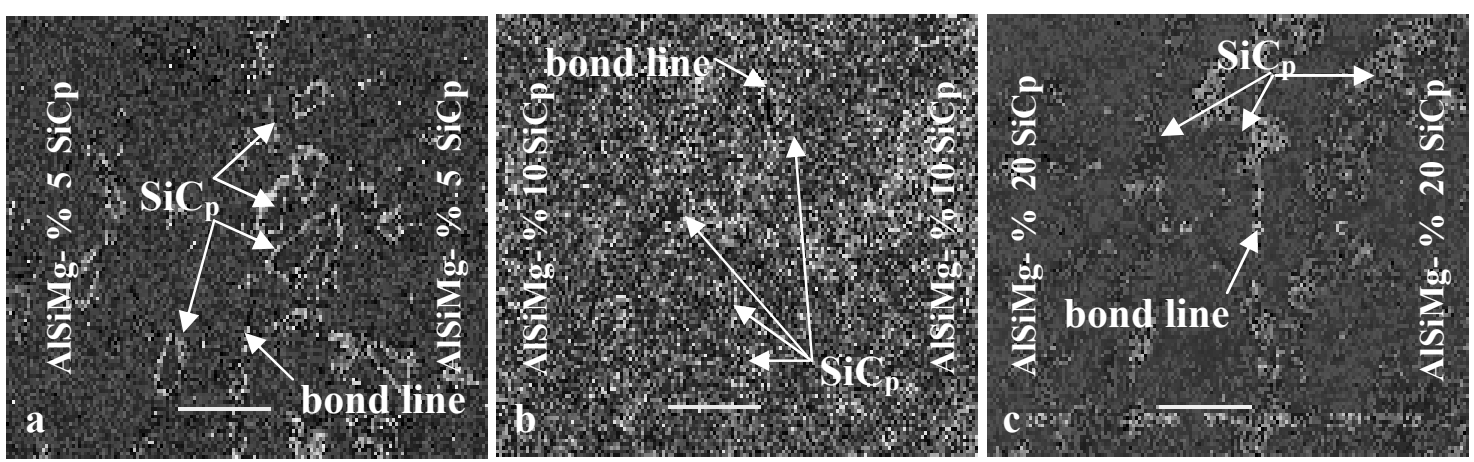

Figure 9. SEM Micrograph of Specimens Bonded at $625^{\circ} \mathrm{C}$ Process Temp. for a-20, b-40, c-60 min

In this study, prediction of microhardness of diffusion bonded MMC couples were performed by using a back-propagation neural network that uses gradient descent learning algorithm.

a- Bonding process temperature, bonding period and SiC particulate (wt) fractions were used as the model inputs while the microhardness was the output of the model. These datas were obtained from experimental works.

b- Comparison of experimental microhardness test results with predicted values inline with bonding parameters were presented in Table 3. Experimental microhardness of specimens have shown a consistency with predicted results differing 0.01-3. This trained values can lead maximum $5 \%$ error in microhardness calculations. 
Table 3. Microhardness of Predicted Values with Actual Values

\begin{tabular}{|c|c|c|c|c|c|c|c|c|c|c|c|c|c|c|c|}
\hline \multirow[t]{2}{*}{$\begin{array}{c}\text { Samp, } \\
\text { No }\end{array}$} & \multirow[t]{2}{*}{$\begin{array}{c}\text { Couples of } \\
\text { samples }\end{array}$} & \multirow[t]{2}{*}{$\begin{array}{l}\text { Temp. } \\
\left({ }^{\circ} \mathrm{C}\right)\end{array}$} & \multirow[t]{2}{*}{$\begin{array}{l}\text { Duratı- } \\
\text { ons(dk.) }\end{array}$} & & $\begin{array}{l}\text { ral valu } \\
\text { rohard }\end{array}$ & & & $\begin{array}{l}\text { icted va } \\
\text { crohard }\end{array}$ & $\begin{array}{l}\text { s of } \\
\text { ss }\end{array}$ & & $\operatorname{rror}(\mathrm{M}$ & & & $\% \operatorname{Err}$ & \\
\hline & & & & $\mathbf{H}_{1}$ & $\mathbf{H}_{2}$ & $\mathbf{H}_{3}$ & $\mathbf{H}_{1}$ & $\mathbf{H}_{2}$ & $\mathbf{H}_{3}$ & $\mathbf{H}_{1}$ & $\mathbf{H}_{2}$ & $\mathbf{H}_{3}$ & $\mathbf{H}_{1}$ & $\mathbf{H}_{2}$ & $\mathbf{H}_{3}$ \\
\hline 1 & $\% 5-5 \mathrm{SiC}_{\mathrm{p}}$ & 550 & 20 & 61.7 & 69.9 & 66.0 & 52.3 & 73.0 & 59.4 & +9.4 & -3.1 & +6.6 & +15.2 & -4.43 & +10.0 \\
\hline 2 & $\%$ 5-10 $\mathrm{SiC}_{\mathrm{p}}$ & 550 & 20 & 71.2 & 70.0 & 65.6 & 80.0 & 76.3 & 55.2 & -8.8 & -6.3 & +10.4 & -12.3 & -9.00 & +15.8 \\
\hline 3 & $\%$ 5-20 $\mathrm{SiC}_{\mathrm{p}}$ & 550 & 20 & 67.6 & 74.0 & 71.4 & 60.5 & 77.7 & 75.0 & +7.1 & -3.7 & -3.6 & +10.5 & -5.00 & -5.04 \\
\hline 4 & $\%$ 10-10 $\mathrm{SiC}_{\mathrm{p}}$ & 550 & 20 & 66.5 & 77.0 & 72.3 & 64.2 & 75.8 & 70.0 & +2.3 & +2.8 & +2.3 & +3.45 & +3.63 & +3.18 \\
\hline 5 & $\% 10-20 \mathrm{SiC}_{\mathrm{p}}$ & 550 & 20 & 67.1 & 78.0 & 74.2 & 71.4 & 72.8 & 76.0 & -4.3 & +5.2 & -1.8 & -6.40 & +6.66 & -2.42 \\
\hline 6 & $\%$ 20-20 $\mathrm{SiC}_{\mathrm{p}}$ & 550 & 20 & 70.5 & 80.0 & 77.2 & 68.5 & 86.6 & 74.7 & +2.0 & -6.6 & +2.5 & +2.83 & -8.25 & +3.23 \\
\hline 7 & $\%$ 5-5 $\mathrm{SiC}_{\mathrm{p}}$ & 550 & 40 & 71.2 & 79.0 & 74.8 & 68.0 & 77.5 & 77.9 & +3.2 & +1.5 & -3.1 & +4.49 & +1.89 & -4.14 \\
\hline 8 & $\%$ 5-10 $\mathrm{SiC}_{\mathrm{p}}$ & 550 & 40 & 67.0 & 81.0 & 73.6 & 73.0 & 74.3 & 70.0 & -6.0 & +6.7 & +3.6 & -8.95 & +8.27 & +4.89 \\
\hline 9 & $\%$ 5-20 $\mathrm{SiC}_{\mathrm{p}}$ & 550 & 40 & 69.3 & 83.0 & 72.2 & 72.7 & 88.8 & 75.9 & -3.4 & -5.8 & -3.7 & -4.90 & -6.98 & -5.12 \\
\hline 10 & $\% \mathbf{1 0 - 1 0} \mathrm{SiC}_{\mathrm{p}}$ & 550 & 40 & 76.8 & 85.0 & 78.6 & 80.0 & 81.6 & 82.3 & -3.2 & +3.4 & -3.7 & -4.16 & +4.00 & -4.70 \\
\hline 11 & $\% \mathbf{1 0}^{-20} \mathrm{SiC}_{\mathrm{p}}$ & 550 & 40 & 80.0 & 87.2 & 81.5 & 73.9 & 90.0 & 78.4 & +6.1 & -2.8 & +3.1 & +7.62 & -3.21 & +3.80 \\
\hline 12 & $\%$ 20-20 $\mathrm{SiC}_{\mathrm{p}}$ & 550 & 40 & 82.9 & 90.0 & 85.3 & 79.3 & 87.1 & 82.0 & +3.6 & +2.9 & +3.3 & +4.34 & +3.22 & +3.86 \\
\hline 13 & $\% 5-5 \mathrm{SiC}_{\mathrm{p}}$ & 550 & 60 & 85.7 & 91.0 & 86.7 & 88.0 & 96.2 & 90.3 & -2.3 & +5.2 & -3.6 & -2.68 & +5.71 & -4.15 \\
\hline 14 & $\%$ 5-10 $\mathrm{SiC}_{\mathrm{p}}$ & 550 & 60 & 88.2 & 94.0 & 92.4 & 84.7 & 90.0 & 89.5 & +3.5 & +4.0 & +2.9 & +3.96 & +4.25 & +3.13 \\
\hline 15 & $\%$ 5-20 $\mathrm{SiC}_{\mathrm{p}}$ & 550 & 60 & 87.8 & 92.0 & 88.7 & 83.8 & 95.8 & 90.7 & +4.0 & -3.8 & -2.0 & +4.55 & -4.13 & -2.25 \\
\hline 16 & $\%$ 10-10 $\mathrm{SiC}_{\mathrm{p}}$ & 550 & 60 & 88.3 & 95.0 & 94.4 & 84.0 & 93.6 & 92.0 & +4.3 & +1.4 & +2.4 & +4.86 & +1.47 & +2.54 \\
\hline 19 & $\% 5-5 \mathrm{SiC}_{\mathrm{p}}$ & 575 & 20 & 87.0 & 92.0 & 84.5 & 85.0 & 90.4 & 80.4 & +3.0 & +1.6 & +4.1 & +3.44 & +1.73 & +4.85 \\
\hline 20 & $\% 5-10 \mathrm{SiC}_{\mathrm{p}}$ & 575 & 20 & 86.9 & 93.0 & 92.4 & 88.7 & 91.9 & 95.3 & -1.8 & +1.1 & -2.9 & -2.07 & +1.18 & -0.31 \\
\hline 21 & $\%$ 5-20 $\mathrm{SiC}_{\mathrm{p}}$ & 575 & 20 & 84.3 & 95.0 & 93.4 & 82.3 & 91.8 & 90.0 & +2.0 & +3.2 & +3.4 & +2.37 & +3.36 & +0.36 \\
\hline 22 & $\%$ 10-10 $\mathrm{SiC}_{\mathrm{p}}$ & 575 & 20 & 89.2 & 97.0 & 89.9 & 94.6 & 93.7 & 86.6 & -5.4 & +3.3 & +3.3 & -6.05 & +3.40 & +3.67 \\
\hline 23 & $\% \mathbf{1 0}^{-20 \mathrm{SiC}_{\mathrm{p}}}$ & 575 & 20 & 89.8 & 99.0 & 97.0 & 85.7 & 96.2 & 100.9 & +4.1 & +2.8 & -3.9 & +4.56 & +2.82 & -4.02 \\
\hline 24 & $\%$ 20-20 $\mathrm{SiC}_{\mathrm{p}}$ & 575 & 20 & 99.0 & 105.0 & 97.1 & 100.2 & 107.8 & 94.7 & -1.2 & -2.8 & +2.4 & -1.21 & -2.66 & +2.47 \\
\hline 25 & $\%$ 5-5 $\mathrm{SiC}_{\mathrm{p}}$ & 575 & 40 & 91.2 & 94.0 & 91.1 & 88.2 & 90.9 & 89.4 & +3.0 & +3.1 & +1.7 & +3.28 & +0.34 & +1.86 \\
\hline 26 & $\% 5-10 \mathrm{SiC}_{\mathrm{p}}$ & 575 & 40 & 89.1 & 96.0 & 92.6 & 90.4 & 99.0 & 89.8 & -1.3 & -3.0 & +2.8 & -1.45 & -3.12 & +3.02 \\
\hline 27 & $\% 5-20 \mathrm{SiC}_{\mathrm{p}}$ & 575 & 40 & 89.3 & 97.0 & 92.5 & 86.6 & 94.5 & 94.0 & +2.7 & +2.5 & -1.5 & +3.02 & +2.57 & -1.62 \\
\hline 28 & $\%$ 10-10 $\mathrm{SiC}_{\mathrm{p}}$ & 575 & 40 & 92.4 & 99.0 & 92.8 & 88.1 & 95.9 & 87.0 & +4.3 & +3.1 & +5.8 & +4.65 & +3.13 & +6.25 \\
\hline 29 & $\% \mathbf{1 0 - 2 0 S i C}_{\mathrm{p}}$ & 575 & 40 & 94.0 & 101.0 & 95.7 & 96.3 & 103.6 & 98.6 & -2.3 & +7.4 & -2.9 & -2.44 & +7.32 & -3.03 \\
\hline 30 & $\%$ 20-20 $\mathrm{SiC}_{\mathrm{p}}$ & 575 & 40 & 94.2 & 103.0 & 98.6 & 91.7 & 100.0 & 95.2 & +2.5 & +3.0 & +3.4 & +2.65 & +2.91 & +3.44 \\
\hline 31 & $\% 5-5 \mathrm{SiC}_{\mathrm{p}}$ & 575 & 60 & 84.4 & 97.0 & 91.5 & 80.0 & 92.9 & 89.8 & +4.4 & +4.1 & +1.7 & +5.21 & +4.22 & +1.85 \\
\hline 32 & $\%$ 5-10 $\mathrm{SiC}_{\mathrm{p}}$ & 575 & 60 & 88.2 & 99.0 & 95.0 & 90.0 & 96.4 & 96.6 & -1.8 & +2.6 & -1.6 & -2.04 & +2.62 & -1.68 \\
\hline 33 & $\% 5-20 \mathrm{SiC}_{\mathrm{p}}$ & 575 & 60 & 88.9 & 100.0 & 94.2 & 91.3 & 102.4 & 90.9 & -2.4 & -2.4 & +3.3 & -2.69 & -2.40 & +3.50 \\
\hline 34 & $\% \mathbf{1 0 - 1 0} \mathrm{SiC}_{\mathrm{p}}$ & 575 & 60 & 93.8 & 103.0 & 94.9 & 90.2 & 100.2 & 91.7 & +3.6 & +2.8 & +3.2 & +3.83 & +2.71 & +3.37 \\
\hline 35 & $\% 10-20 \mathrm{SiC}_{\mathrm{p}}$ & 575 & 60 & 93.7 & 104.0 & 101.4 & 95.0 & 106.4 & 105.0 & -1.3 & -2.4 & -3.6 & -1.38 & -2.30 & -3.55 \\
\hline 36 & $\%$ 20-20 $\mathrm{SiC}_{\mathrm{p}}$ & 575 & 60 & 97.7 & 108.0 & 102.3 & 100.0 & 110.5 & 104.7 & -2.3 & -2.5 & -2.4 & -2.35 & -2.31 & -2.34 \\
\hline 39 & $\% 5-20 \mathrm{SiC}_{\mathrm{p}}$ & 600 & 20 & 94.3 & 104.0 & 100.0 & 90.4 & 99.6 & 92.8 & +3.9 & +4.4 & +7.2 & +4.13 & +4.23 & +7.20 \\
\hline 40 & $\% \mathbf{1 0 - 1 0} \mathrm{SiC}_{\mathrm{p}}$ & 600 & 20 & 94.3 & 107.0 & 99.6 & 86.6 & 108.3 & 95.7 & +7.7 & -1.3 & +3.9 & +8.16 & -1.21 & +3.91 \\
\hline 41 & $\% 10-20 \mathrm{SiC}_{\mathrm{p}}$ & 600 & 20 & 100.2 & 109.0 & 104.2 & 96.8 & 111.4 & 100.5 & +3.4 & -2.4 & +3.7 & +3.39 & -2.20 & +3.55 \\
\hline 42 & $\%$ 20-20 $\mathrm{SiC}_{\mathrm{p}}$ & 600 & 20 & 100.5 & 111.0 & 105.3 & 91.9 & 108.6 & 101.7 & +8.6 & +2.4 & +3.6 & +8.55 & +2.16 & +3.41 \\
\hline 43 & $\% 5-5 \mathrm{SiC}_{\mathrm{p}}$ & 600 & 40 & 93.3 & 104.0 & 94.4 & 90.0 & 100.8 & 90.9 & +3.3 & +3.2 & +3.5 & +3.53 & +3.07 & +3.70 \\
\hline 44 & $\%$ 5-10 $\mathrm{SiC}_{\mathrm{p}}$ & 600 & 40 & 94.0 & 105.0 & 99.2 & 91.6 & 101.3 & 94.9 & +2.4 & +3.7 & +4.3 & +2.55 & +0.25 & +4.33 \\
\hline 45 & $\%$ 5-20 $\mathrm{SiC}_{\mathrm{p}}$ & 600 & 40 & 96.2 & 107.0 & 102.9 & 92.5 & 104.7 & 100.0 & +3.7 & +2.3 & +2.9 & +3.84 & +2.14 & +2.81 \\
\hline 46 & $\% \mathbf{1 0 - 1 0} \mathrm{SiC}_{\mathrm{p}}$ & 600 & 40 & 98.6 & 110.0 & 103.3 & 100.0 & 112.7 & 99.7 & -1.4 & -2.7 & +3.6 & -1.41 & -2.45 & +3.48 \\
\hline 47 & $\% 10-20 \mathrm{SiC}_{\mathrm{p}}$ & 600 & 40 & 100.2 & 108.0 & 102.6 & 103.6 & 110.9 & 99.8 & -3.4 & -2.9 & +2.8 & -3.39 & -2.68 & +2.72 \\
\hline 48 & $\%$ 20-20 $\mathrm{SiC}_{\mathrm{p}}$ & 600 & 40 & 103.3 & 113.0 & 105.7 & 106.2 & 109.8 & 109.6 & -2.9 & +3.2 & -3.9 & -2.80 & +2.83 & -3.68 \\
\hline 49 & $\% 5-5 \mathrm{SiC}_{\mathrm{p}}$ & 600 & 60 & 96.8 & 107.0 & 101.9 & 99.7 & 110.4 & 104.0 & -2.9 & -3.4 & -2.1 & -2.99 & -3.17 & -2.06 \\
\hline 50 & $\%$ 5-10 $\mathrm{SiC}_{\mathrm{p}}$ & 600 & 60 & 97.2 & 108.0 & 100.9 & 93.8 & 104.7 & 96.6 & +3.4 & +3.3 & +4.3 & +3.49 & +3.05 & +4.26 \\
\hline 51 & $\% 5-20 \mathrm{SiC}_{\mathrm{p}}$ & 600 & 60 & 100.5 & 110.0 & 106.1 & 92.7 & 100.0 & 101.2 & +7.8 & +10.0 & +3.9 & +7.76 & +9.09 & +3.67 \\
\hline 52 & $\%$ 10-10 $\mathrm{SiC}_{\mathrm{p}}$ & 600 & 60 & 104.3 & 110.0 & 103.1 & 100.7 & 103.8 & 100.0 & +3.7 & +6.2 & +3.1 & +3.54 & +5.63 & +3.01 \\
\hline 53 & $\% \mathbf{1 0 - 2 0} \mathrm{SiC}_{\mathrm{p}}$ & 600 & 60 & 105.6 & 113.0 & 110.1 & 99.9 & 110.2 & 114.2 & +5.7 & +2.8 & -4.1 & +5.39 & +2.47 & -3.72 \\
\hline 54 & $\%$ 20-20 $\mathrm{SiC}_{\mathrm{p}}$ & 600 & 60 & 108.6 & 116.0 & 108.2 & 110.4 & 120.8 & 104.4 & -1.8 & -4.8 & +3.8 & -1.65 & -4.13 & +3.51 \\
\hline 55 & $\% 5-5 \mathrm{SiC}_{\mathrm{p}}$ & 625 & 20 & 96.9 & 110.0 & 103.5 & 92.2 & 103.3 & 98.8 & +3.3 & -3.3 & +4.7 & +3.40 & -3.00 & +4.54 \\
\hline 56 & $\%$ 5-10 $\mathrm{SiC}_{\mathrm{p}}$ & 625 & 20 & 98.6 & 111.0 & 107.7 & 93.6 & 104.6 & 100.3 & +5.0 & +6.4 & +7.4 & +5.07 & +5.76 & +6.87 \\
\hline $\mathbf{5 7}$ & $\%$ 5-20 $\mathrm{SiC}_{\mathrm{p}}$ & 625 & 20 & 101.9 & 113.0 & 110.3 & 92.5 & 110.4 & 107.8 & +9.4 & +2.6 & +2.5 & +9.22 & +2.30 & +2.26 \\
\hline 58 & $\% \mathbf{1 0 - 1 0} \mathrm{SiC}_{\mathrm{p}}$ & 625 & 20 & 100.0 & 114.0 & 108.7 & 97.2 & 108.0 & 104.9 & +2.8 & +6.0 & +3.8 & +2.80 & +5.26 & +3.49 \\
\hline 59 & $\% \mathbf{1 0 - 2 0 S i C}_{\mathrm{p}}$ & 625 & 20 & 103.6 & 112.0 & 109.1 & 99.6 & 108.9 & 105.7 & +4.0 & +3.1 & +3.7 & +3.86 & +2.76 & +3.39 \\
\hline 60 & $\% \mathbf{2 0 - 2 0} \mathrm{SiC}_{\mathrm{p}}$ & 625 & 20 & 109.9 & 118.0 & 113.0 & 112.8 & 113.6 & 115.4 & -2.9 & +4.4 & -2.4 & -2.63 & +3.72 & -2.12 \\
\hline 61 & $\%$ 5-5 $\mathrm{SiC}_{\mathrm{p}}$ & 625 & 40 & 100.6 & 112.0 & 105.1 & 105.8 & 114.6 & 107.2 & -5.2 & -2.6 & -2.1 & -5.16 & -2.32 & -1.99 \\
\hline 62 & $\% 5-10 \mathrm{SiC}_{\mathrm{p}}$ & 625 & 40 & 104.6 & 114.0 & 109.1 & 101.1 & 109.8 & 104.6 & +3.5 & +4.2 & +4.5 & +3.34 & +3.68 & +4.12 \\
\hline 63 & $\% 5-20 \mathrm{SiC}_{\mathrm{p}}$ & 625 & 40 & 106.6 & 116.0 & 107.6 & 100.0 & 112.3 & 105.0 & +6.6 & +3.7 & +2.6 & +6.19 & +3.18 & +2.41 \\
\hline 64 & $\%$ 10-10 $\mathrm{SiC}_{\mathrm{p}}$ & 625 & 40 & 108.0 & 117.0 & 109.0 & 104.6 & 113.9 & 104.9 & +3.4 & +3.1 & +4.1 & +3.14 & +2.64 & +3.76 \\
\hline 65 & $\% 10-20 \mathrm{SiC}_{\mathrm{p}}$ & 625 & 40 & 109.5 & 115.0 & 109.1 & 107.2 & 110.3 & 106.1 & +2.3 & +4.7 & +3.0 & +2.10 & +4.08 & +2.74 \\
\hline 66 & $\%$ 20-20 $\mathrm{SiC}_{\mathrm{p}}$ & 625 & 40 & 112.6 & 118.0 & 113.2 & 110.0 & 116.4 & 109.8 & +2.6 & +1.6 & +3.4 & +2.30 & +1.35 & +3.01 \\
\hline 67 & $\%$ 5-5 $\mathrm{SiC}_{\mathrm{p}}$ & 625 & 60 & 107.0 & 115.0 & 107.9 & 109.4 & 112.0 & 110.0 & -2.4 & +3.0 & -2.1 & -2.24 & +2.60 & -1.94 \\
\hline 68 & $\% 5-10 \mathrm{SiC}_{\mathrm{p}}$ & 625 & 60 & 106.2 & 117.0 & 107.9 & 103.4 & 114.6 & 103.7 & +2.8 & +2.4 & +4.2 & +2.63 & +2.05 & +3.89 \\
\hline 69 & $\%$ 5-20 $\mathrm{SiC}_{\mathrm{p}}$ & 625 & 60 & 106.0 & 119.0 & 112.2 & 108.5 & 121.2 & 115.0 & -2.5 & -2.2 & -2.8 & -0.23 & -1.84 & -2.49 \\
\hline 70 & $\%$ 10-10 $\mathrm{SiC}_{\mathrm{p}}$ & 625 & 60 & 112.3 & 120.0 & 112.5 & 110.0 & 117.3 & 110.6 & +2.3 & +2.7 & +1.9 & +2.04 & +2.25 & +1.68 \\
\hline 71 & $\% 10-20 \mathrm{SiC}_{\mathrm{p}}$ & 625 & 60 & 114.0 & 120.0 & 117.8 & 111.8 & 118.1 & 115.6 & +2.2 & +1.9 & +2.2 & +1.92 & +1.58 & +1.86 \\
\hline 72 & $\%$ 20-20 $\mathrm{SiC}_{\mathrm{p}}$ & 625 & 60 & 114.2 & 122.0 & 117.3 & 110.9 & 117.9 & 114.4 & +3.3 & +4.1 & +2.9 & +2.88 & +3.36 & +2.47 \\
\hline
\end{tabular}


c- The Sum-squared error (SSE) graphic trained for 623500 Epochs was presented in Fig.11.

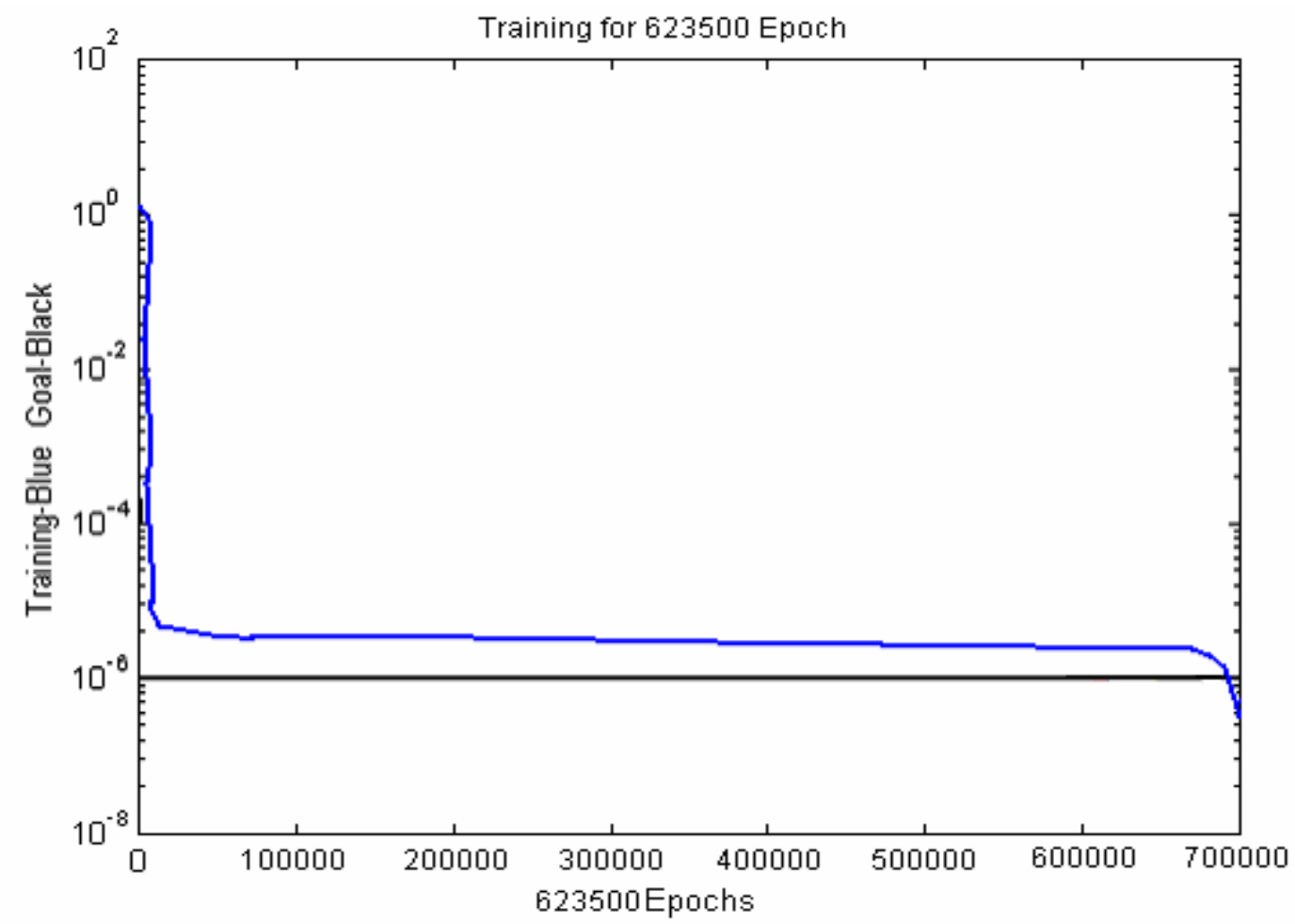

Figure 11. Sum-Squared Error Curve Versus İteration Number

\section{CONCLUSIONS}

The overall performance of the model was quite satisfactory. The low error fractions indicate that ANNs could be a useful tool for modeling and predicting microhardness of bonded interfaces of $\mathrm{SiC}_{\mathrm{p}}$ reinforced $\mathrm{Al}$ alloy MMCs. Under given conditions, and with prescribed materials predicted microhardness can be utilized by designers and process engineers as and where necessary. Given and predicted values of the ANN system can also be employed at feasibility programs at no cost. This can be handled as a cost saving item at advanced production planning.

\section{Acknowledgements}

The authors are gratefull to Firat University Research committee since this study is suggested and supported through grant (FÜBAP -983). 


\section{REFERENCES}

1- Koker, R., Altinkok, N., Modelling of The Prediction of Tensile and Density Properties in Particle Reinforced Metal Matrix Composites by Using Neural Networks, Materials and Design, 1-7, 2005.

2- Altinkok, N. Koker, R., Neural Network Approach to Prediction of Bending Strength and Hardening Behaviour of Particulate Reinforced (Al-Si-Mg)-Aluminium Matrix Composites, Materials and Design, 25, 595-602, 2005.

3- Avci, E., Turkoglu I., and Poyraz, M., Intelligent Target Recognition on Based Wavelet Packet Neural Network", Elsevier Expert Systems with Applications, 29, 175$182,2005$.

4- Avci, E., Turkoglu I., and Poyraz, M., Intelligent Target Recognition Based on Wavelet Adaptive Network Based Fuzzy Inference System, Lecture Notes in Computer Science, Springer-Verlag, 3522, 594-601, 2005.

5- Chun, M.S., Biglou, J., Lenard, J.G., and Kim, J.G., Using Neural Networks to Predict Parameters in The Hot Working of Aluminum Alloys, Journal of Materials Processing Technology, 86, 245-25, 1999.

6- Ganesan,G., Raghukandan, K., Karthikeyan, R. and Pai,B.C., Development of Processing Map for 6061 Al/15\% SiCp Through Neural Networks, Journal of Materials Processing Technology, 166, 423-429, 2005.

7- Jalham, I.S., A comparative study of some network approaches to predict the effect of the reinforcement content on the hot strength of Al-base composites, Journal of Materials Processing Technology,166, 392-397, 2005.

8- Li, H. J., Qi, L.H., Han, H.M.and Guo, L.J., Neural Network Modeling and Optimization of Semi-Solid Extrusion for Aluminum Matrix Composites, Journal of Materials Processing Technology, 151, 126-132, 2004.

9- Caligulu, U., The Investigation of Joinability of Diffusion Bonding with Hot Pressing Manifactured AlSiMg-SiC $p$ Reinforced Composites, Firat Uni. Graduate School of Naturel and Applied Sciences Department of Metallurgy Education, Master Thesis, Elazig, 2005.

10- Taskin, M., Diffusion bonding of fine grained high carbon steels in the superplasticity temperature range, Firat University Graduate School of Naturel and Applied Sciences Department of Metallurgy Education, PhD Thesis, Elazig,2000.

11- Anijdan, S.H.M., Bahrami, A., Hosseini, H.R.M., and Shafyei, A., Using genetic algorithm and artificial neural network analyses to design an Al-Si casting alloy of minimum porosity, Materials and Design, 2005.

12- Okuyucu, H., Kurt, A., and Arcaklioglu, E., Artificial neural network application to the friction stir welding of aluminum plates, Materials and Design, 2005. 